\title{
TV/Series
}

9| 2016

Guerres en séries (I)

\section{Contre le split-screen. Autopsie d'un artifice}

\section{Ophir Lévy}

\section{(2) OpenEdition}

Journals

Édition électronique

URL : http://journals.openedition.org/tvseries/1302

DOI : 10.4000/tvseries.1302

ISSN : 2266-0909

\section{Éditeur}

GRIC - Groupe de recherche Identités et Cultures

\section{Référence électronique}

Ophir Lévy, «Contre le split-screen. Autopsie d'un artifice », TV/Series [En ligne], 9 | 2016, mis en ligne le 01 juin 2016, consulté le 14 novembre 2019. URL : http://journals.openedition.org/tvseries/1302 ; DOI : $10.4000 /$ tvseries. 1302

Ce document a été généré automatiquement le 14 novembre 2019.

\section{(c) (i) () $\Theta$}

TV/Series est mis à disposition selon les termes de la licence Creative Commons Attribution - Pas d'Utilisation Commerciale - Pas de Modification 4.0 International. 


\title{
Contre le split-screen. Autopsie d'un artifice
}

\author{
Ophir Levy
}

1 Très en vogue depuis la fin des années 1960 , le procédé du split-screen, consistant à diviser le cadre filmique en plusieurs portions, compte parmi les figures stylistiques les plus marquantes de 24 heures chrono (Fox, 2001-2014). Au point que la série s'impose aujourd'hui comme un exemple incontournable dès lors qu'il s'agit d'illustrer ce procédé technique ${ }^{1}$. Or, par-delà la dimension de signature visuelle que revêt ici le split-screen voire d'« habillage », au sens le plus platement télévisuel du terme - dans quelle mesure les auteurs de 24 heures chrono parviennent-ils à tirer profit d'une telle démultiplication des écrans? Face au procédé du split-screen, nous avons le même sentiment que celui d'André Bazin vis-à-vis de certaines formes ou de certains usages du montage. Le sentiment, pour le dire trivialement, d'une vague escroquerie de mise en scène dont l'artificialité conduit cependant, en définitive, à s'interroger sur les fondements mêmes de l'écriture cinématographique.

Dans un article célèbre intitulé « Montage interdit », André Bazin lance un pavé dans la mare des partisans du montage-roi en affirmant qu'« il est des cas où, loin de constituer l'essence du cinéma, le montage en est la négation ${ }^{2}$ ». Cette proposition polémique s'appuie chez Bazin sur la foi préalable en une ontologie de l'image photographique et cinématographique - mâtinée de christianisme - à laquelle le montage ferait dans certains cas violence. D'après Bazin, l'essence du cinéma réside dans sa capacité à se faire « empreinte » de la réalité, à en accueillir l'« ambiguïté » et à en restituer la continuité Loin de nous l'idée d'attribuer arbitrairement une quelconque essence à l'image cinématographique. Il ne s'agit ici que de reprendre à notre compte l'intuition bazinienne selon laquelle un simple procédé technique (le montage, le travelling ou, en l'occurrence, le split-screen), suivant l'usage que l'on en fait, peut constituer l'indice d'un certain génie du cinéma ${ }^{4}$. C'est-à-dire de propriétés singulières du cinéma que ces techniques nous révèlent, soit parce qu'elles en permettent le déploiement (le sentiment de la durée offert par le plan-séquence, la sensation archaïque du mouvement suscitée par le travelling) 
soit, au contraire, parce que les limites de ces procédés nous font précisément prendre conscience de ce à quoi nous tenons vraiment au cinéma ${ }^{5}$.

3 Soyons clair. Il n'est pas question de condamner ici le procédé du split-screen en soi (ce qui serait absurde). Ni d'affirmer que tous les usages du split-screen sont voués à ne déboucher que sur des résultats artificiels et médiocres (ce qui serait dogmatique). Il ne s'agit pas non plus d'appeler de nos vœux la disparition du split-screen (ce qui serait stupide). Ce que nous souhaitons critiquer ici dans un premier temps, c'est un certain usage du split-screen, qui nous semble par ailleurs être l'usage dominant de ce procédé, et que l'on retrouve pleinement à l'œuvre dans 24 heures. Toutefois, bien qu'il y soit employé avec sa pauvreté coutumière, nous verrons par la suite comment, en dépit de lui-même et des effets qu'il vise à produire, le split-screen participe malgré tout du plaisir si singulier que procure la série en s'inscrivant dans une forme de sadisme narratif.

\section{Pauvreté du split-screen}

4 Le procédé du split-screen, tel qu'il est le plus souvent utilisé, se résume à une exacerbation boursouflée des modes d'écriture, de production de sens et d'affection du spectateur propres au cinéma. Mais afin de ne pas les stigmatiser de manière indifférenciée, nous proposons tout d'abord de distinguer plusieurs usages du split-screen, cités ici par ordre croissant d'intérêt, de pertinence et de sophistication, tant sur le plan narratif qu'esthétique.

Fig. 1 - L'usage tautologique du split-screen : L'Étrangleur de Boston (1968) de Richard Fleischer

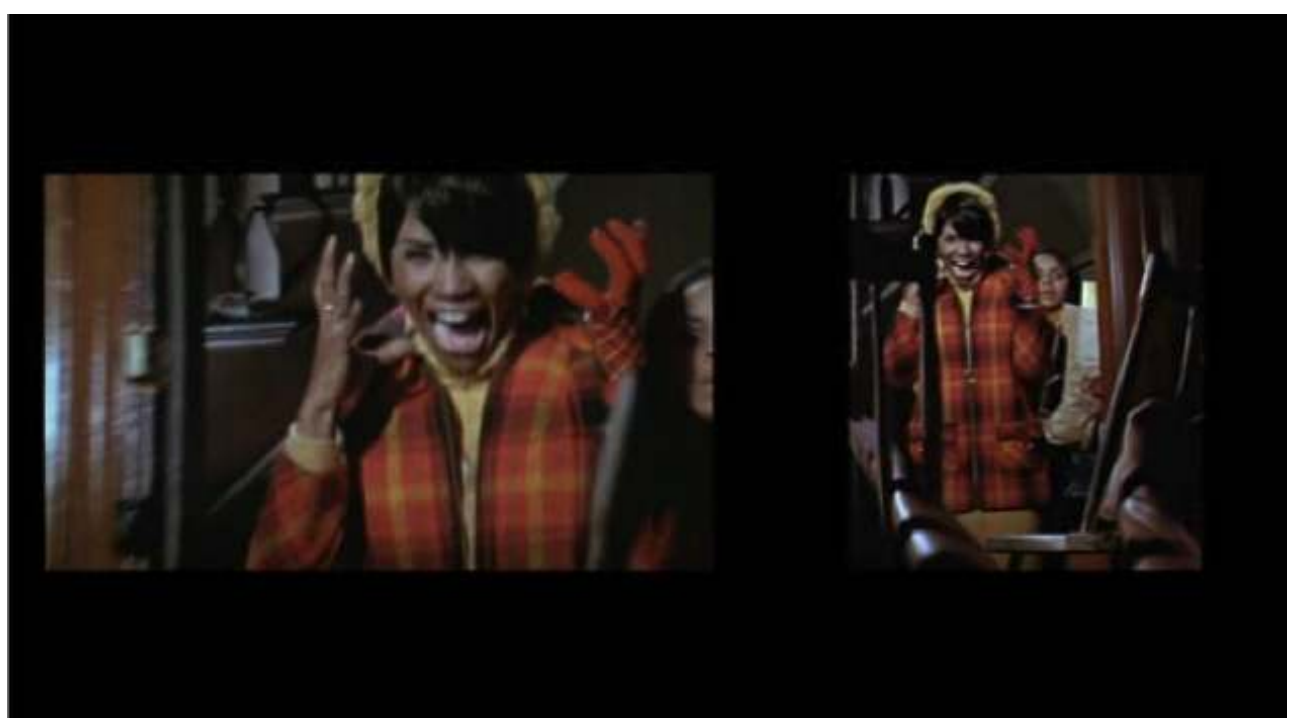

L'usage tautologique consiste à montrer tout bonnement deux fois la même chose (le même objet, le même visage, la même action), filmée à une échelle de plan quasi similaire, mais dans deux portions du cadre différentes [fig. 1-3]. 
Fig. 2 et 3 - L'usage tautologique dans 24 heures chrono (1.5)

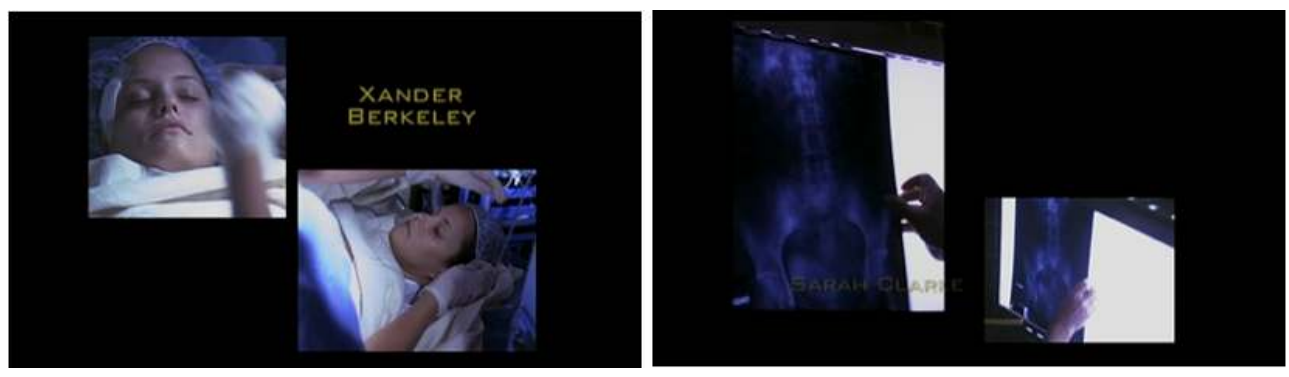

6 L'usage faible ou nivelant : qui consiste à déplier sur l'écran deux actions ou deux points de vue concomitants grâce au split-screen.

Fig. 4 - L'usage faible (le champ contrechamp) : L'Étrangleur de Boston

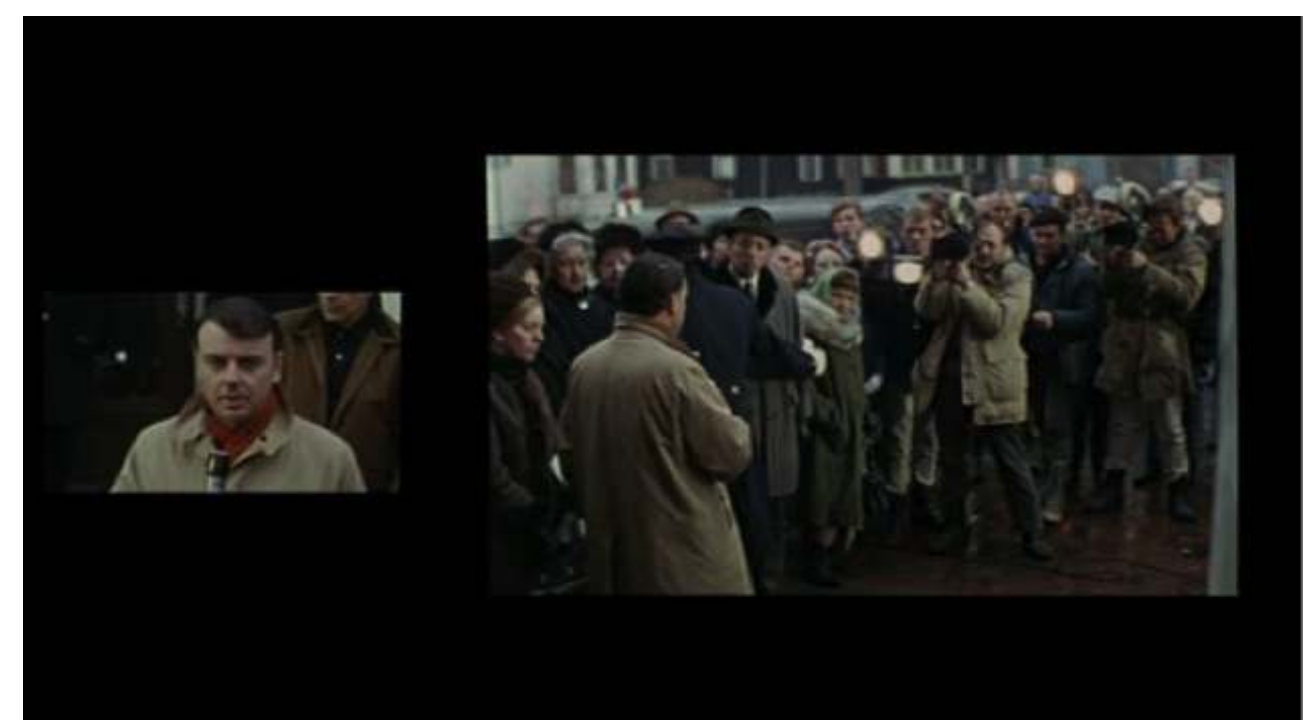

7 Par exemple en le substituant au champ-contrechamp traditionnel [fig. 4], le cas de figure le plus courant étant celui de la conversation téléphonique dont les interlocuteurs apparaissent simultanément à l'image [fig.5-6]. Le split-screen peut également se substituer au montage alterné de deux actions convergentes ou bien au montage parallèle de deux actions disjointes.

Fig. 5 et 6 - L'usage faible (la conversation téléphonique en 1.1 et 1.5)
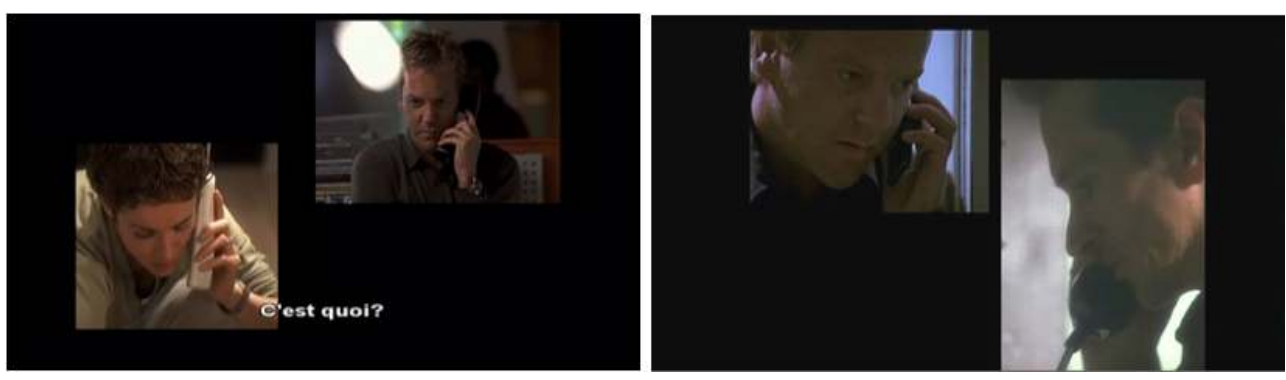

8 L'usage graphique : le split-screen répond souvent à un souci formel, ainsi qu'à une volonté d'exploration des possibilités expressives de l'image. À cet égard, il intéresse au premier 
chef le cinéma d'avant-garde (si l'on songe par exemple aux expérimentations de Dziga Vertov dans L'Homme à la caméra en 1929) et l'art contemporain.

Fig. 7 et 8 - L'usage graphique du split-screen : L'Affaire Thomas Crown (1968) de Norman Jewison
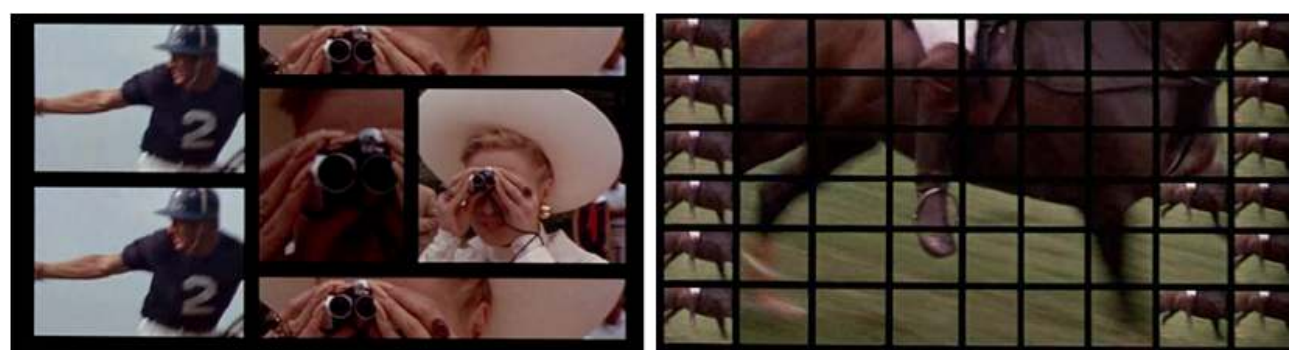

9 Il est important de noter que deux des films les plus emblématiques de l'usage du splitscreen, L'Étrangleur de Boston de Richard Fleischer et L'Affaire Thomas Crown de Norman Jewison [fig. 7-8], sortirent en 1968 et furent tous deux réalisés par des cinéastes ayant assisté, l'année précédente, à l'Exposition universelle de Montréal où furent projetées des œuvres employant un procédé voisin. En l'occurrence, les Diapolyekran de l'artiste tchécoslovaque Josef Svoboda [fig. 9]. 
Fig. 9 - Diapolyekran de Josef Svoboda
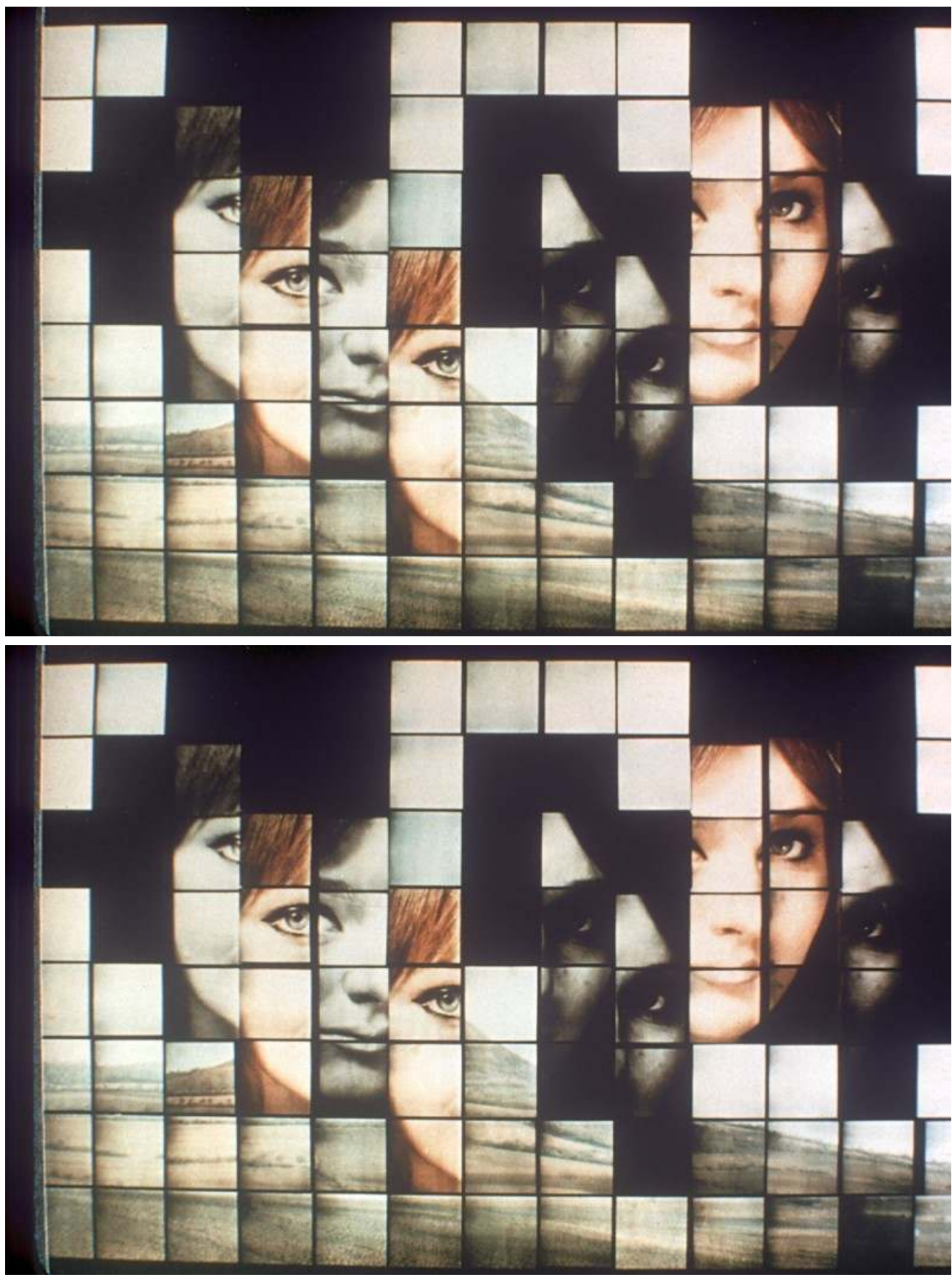

10 À la télévision, le parent pauvre de cette dimension graphique du split-screen est sa fonction d'« habillage ». Habillage vidéo qui, dans 24 heures, par un heureux hasard de la langue française, accompagne régulièrement les scènes au cours desquelles les personnages se vêtissent, endossant du même coup les attributs d'agents spéciaux qui les caractérisent, ou au contraire, dans le cas des terroristes, le costume ordinaire qui les fond dans la masse, le split-screen venant souligner ici leur duplicité [fig. 10]. 
Fig. 10 - Le split-screen comme « habillage » (1.7)

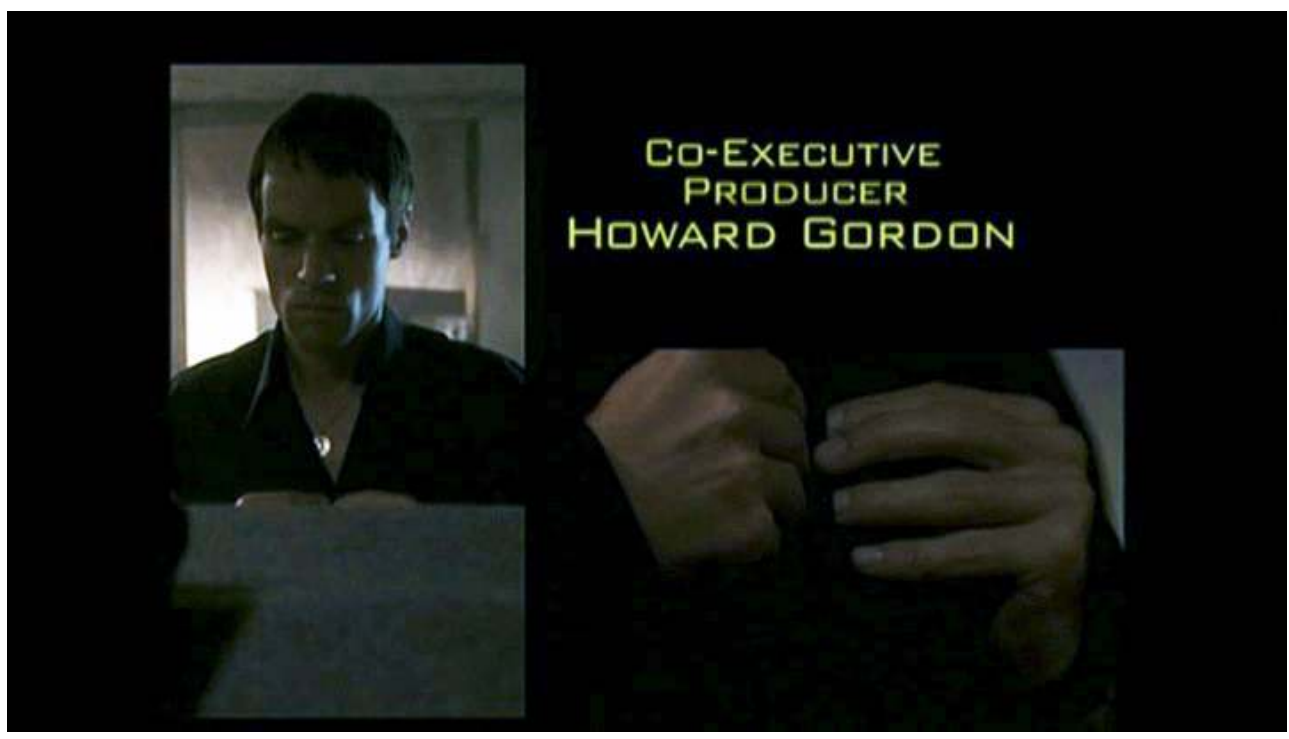

11 L'usage didactique : le split-screen permet, avec plus ou moins de finesse selon les cas, de suggérer, d'affirmer ou de marteler grossièrement les troubles de l'identité, voire le clivage psychique, des personnages.

Fig. 11 - L'usage didactique du split-screen : L'Affaire Thomas Crown

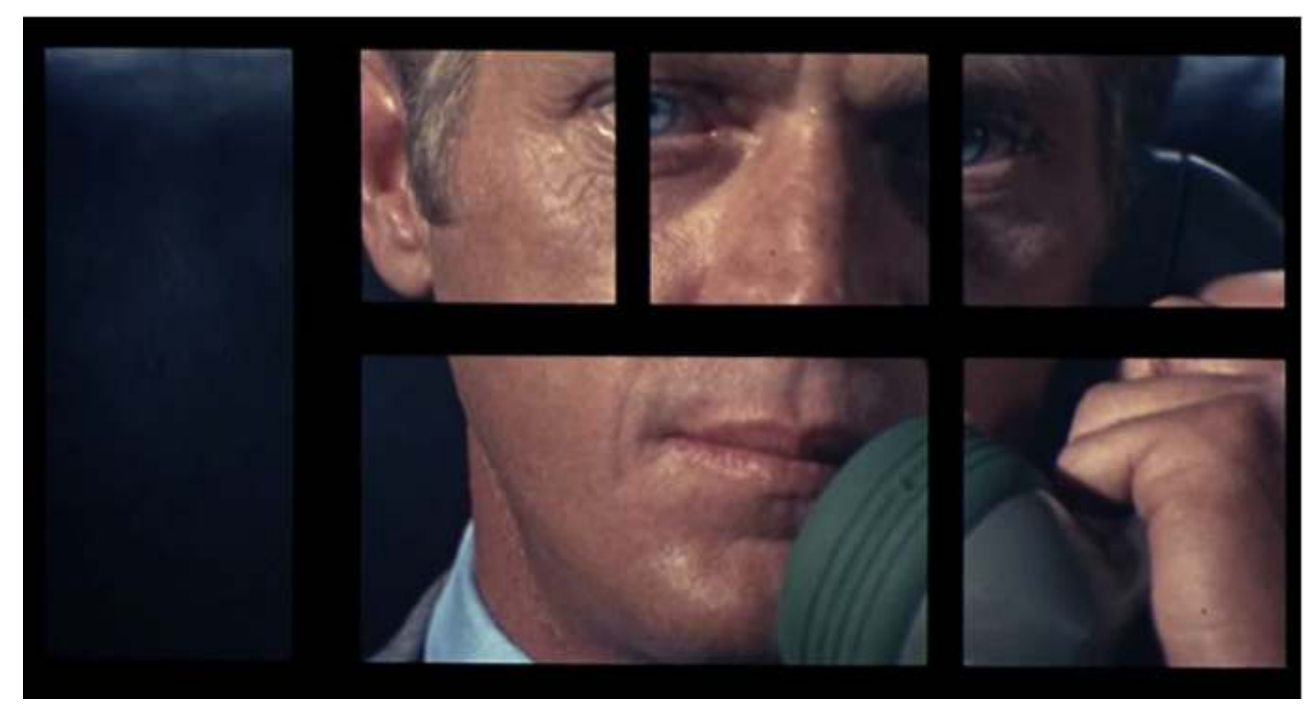

Soit qu'il s'agisse d'une personnalité complexe et mystérieuse dont le film assemble une à une les pièces à l'image d'un puzzle (par exemple dans L'Affaire Thomas Crown de Norman Jewison [fig. 11]), soit qu'il s'agisse d'un personnage schizophrène comme dans L'Étrangleur de Boston ou dans Sisters (1973) de Brian de Palma [fig. 12]. 
Fig. 12 - Sisters (1973) de Brian de Palma : la jointure des portes-miroirs qui fend le visage en deux redouble la schize au sein même du split-screen

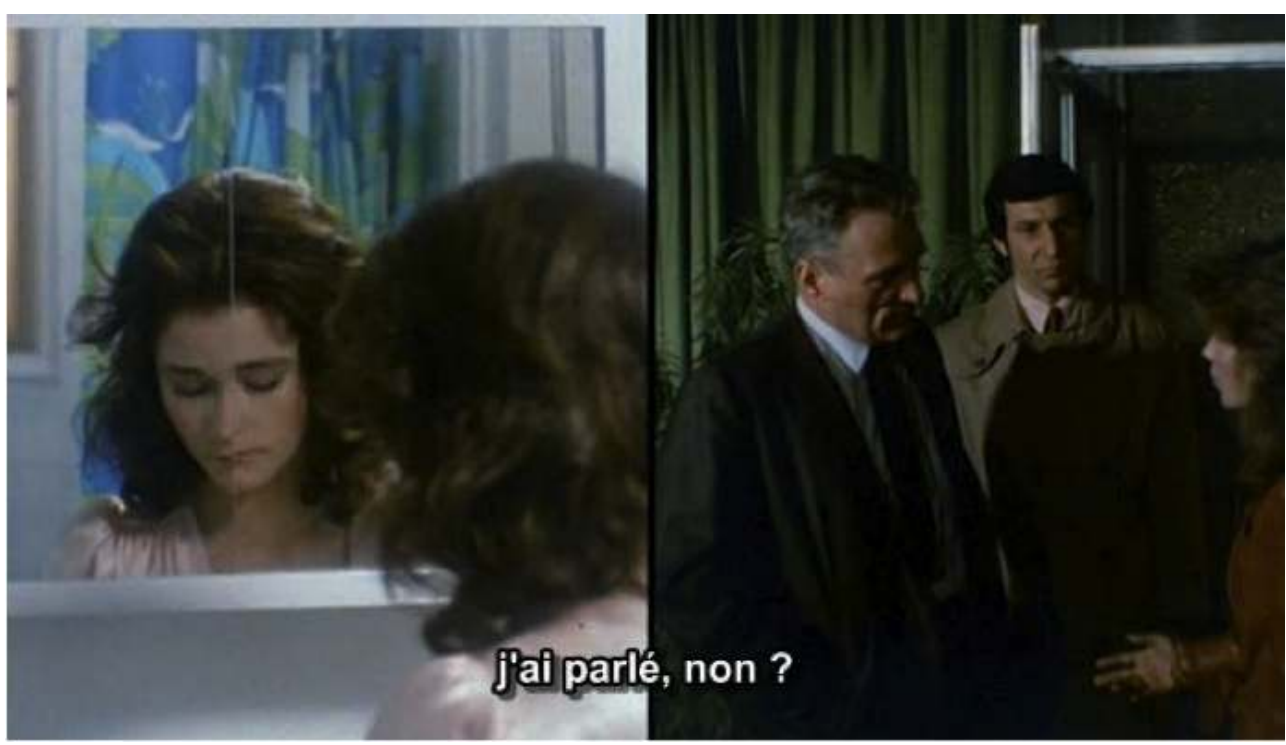

L'usage dialectique : qui consiste à faire du split-screen le révélateur des contradictions ou des antagonismes qui opposent les personnages. Citons l'exemple amusant des deux séances parallèles, chez leurs psychanalystes respectifs, d'Annie et Alvy dans Annie Hall (1977) de Woody Allen. À la même question ("Quelle est la fréquence de vos rapports ?»), les deux amants apportent la même réponse (« trois fois par semaine »). Mais l'accord sur les faits accentue de façon impitoyable le désaccord sur le plan du ressenti. Trois fois par semaine, c'est-à-dire "presque jamais» pour lui, et "constamment» pour elle. La fracture au sein du cadre manifeste donc ici la fracture au sein du couple [fig. 13-14].

Fig. 13-14 - L'usage dialectique : Annie Hall (1977) de Woody Allen
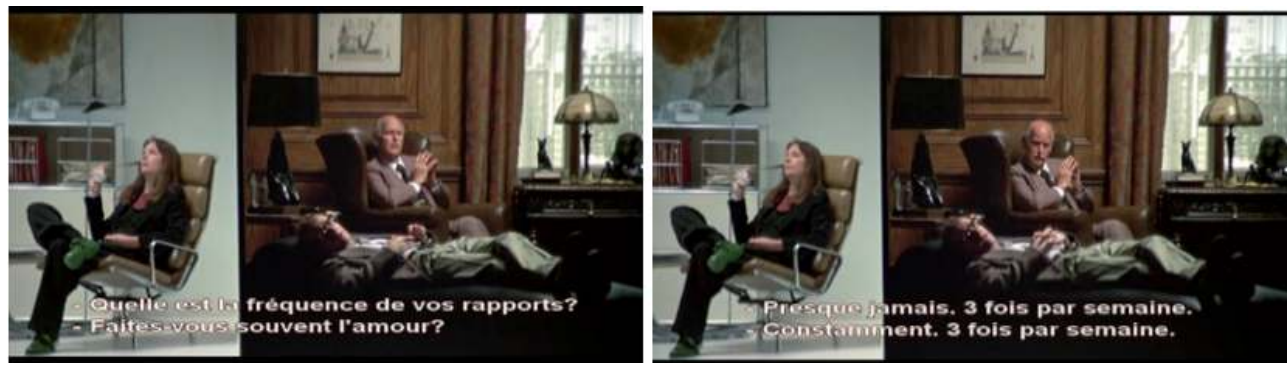

Quels sont les reproches que nous formulons à l'encontre du split-screen ? Et comment révèlent-ils en creux ce à quoi nous tenons au cinéma? Notons tout d'abord que dans la plupart des usages qui sont faits du split-screen, les procédés de montage les plus classiques se trouvent étalés à l'intérieur même du cadre (champ contrechamp, montage alterné, montage parallèle, raccord dans le regard) sans que ne survivent les effets propres à ces types montages: le saisissement, la surprise, l'empreinte laissée sur les images par celles qui les ont précédées, la beauté des raccords ou encore la part d'incertitude liée à la capacité d'association propre à chaque spectateur (le «montage intellectuel », celui qui se fait par la pensée comme l'écrit Eisenstein $\left.{ }^{6}\right)$. Les images cohabitent mais elles ne s'affectent plus. 
Le split-screen induit par ailleurs une forme d'atténuation ou de réduction de la dimension de choix, de renoncement, de deuil, propre au cadrage. Dimension qui, au cinéma, constitue en grande partie le geste artistique lui-même. Bien sûr, il va de soi que le splitscreen relève également d'un choix et même de multiples choix, mais qui se situe davantage du côté de la toute-puissance que de celui du renoncement créateur. D'autre part, le split-screen spatialise le récit à outrance et dissout l'expérience de la durée. Et si, comme le fait le montage, le split-screen peut suggérer au spectateur d'associer différents espaces, différents personnages, différents motifs et différents enjeux narratifs, l'association nous est en revanche mise sous le nez par la coprésence des divers éléments dans un seul et même cadre.

Mais la véritable limite du split-screen est sans doute liée au son. Car s'il fallait synchroniser aux trois ou quatre écrans le mélange d'autant d'environnements sonores simultanés, composés chacun d'une bonne dizaine de pistes son (dialogues, bruitages, musique, son d'ambiance) le résultat serait tout bonnement inaudible et inintelligible. En vérité, lorsqu'il y a split-screen, le regard est presque immanquablement redirigé sur l'un des petits cadres par l'environnement sonore qui prédomine au sein du mixage.

Dédoublement du cadre, mise à plat des procédés de montage, contournement de la dimension de choix, associations et interprétations téléguidées : en somme, et pour le dire de façon brutale, le split-screen, c'est l'écriture cinématographique surlignée au Stabilo Boss !

\section{L'emploi du split-screen dans 24 heures chrono}

18 À quels moments précis, au sein des épisodes de la série, le split-screen est-il systématiquement employé ? En début d'épisode, où il permet de ré-acclimater le téléspectateur à la pluralité des fils narratifs qui sont entremêlés, et où il déballe les pièces du puzzle qui est de nouveau offert au spectateur. C'est en général à ce moment-là que l'usage du split-screen est le plus faible et le plus tautologique. Puis le procédé réapparaît systématiquement après chaque coupure publicitaire, où il redistribue les cartes et réexpose les trames narratives en cours [fig. 15]. 
Fig. 15 - Réexposer les trames narratives (1.2)

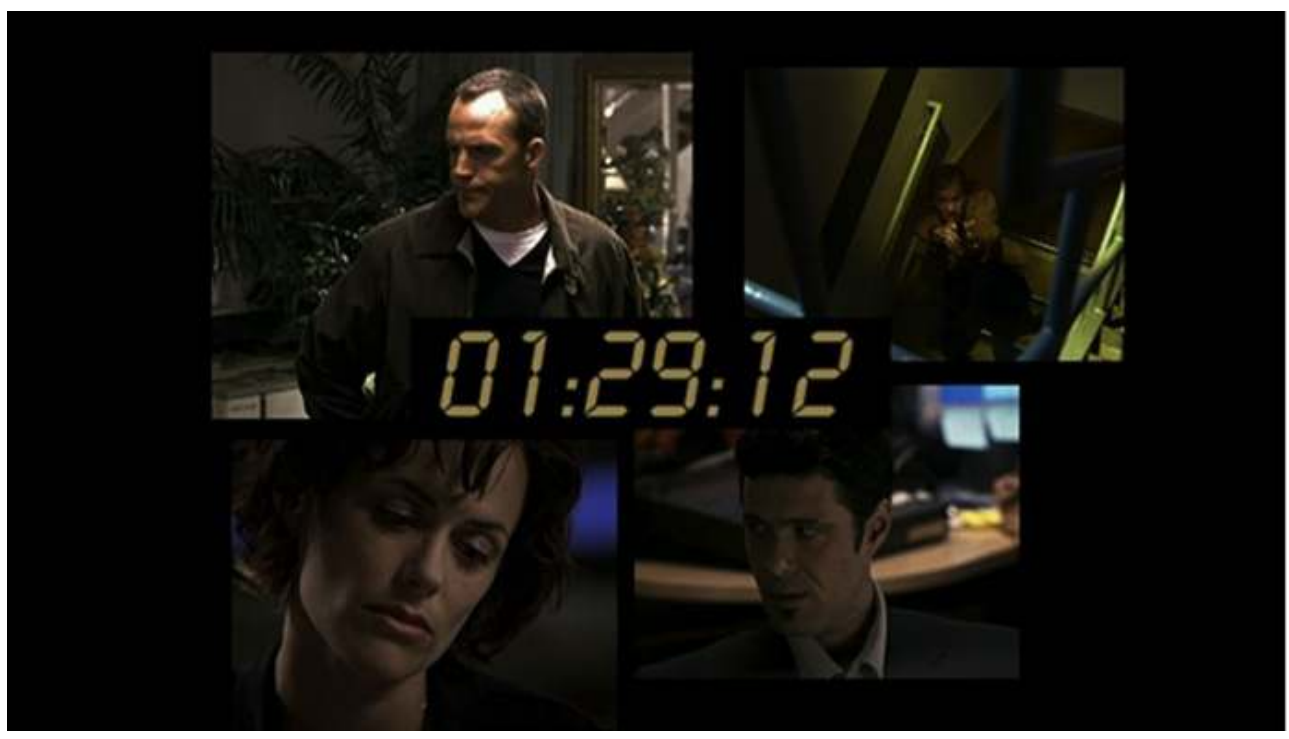

Enfin, lorsque l'épisode se termine, en rassemblant les différentes actions en cours, l'usage du split-screen produit, sur le plan narratif, un effet cette fois-ci très intéressant de suspension, d'ouverture et d'attention à l'égard des possibles. Autant de postures qui, selon Paul Ricœur, devraient être celles de l'historien lorsque ce dernier raconte les événements du passé. L'historien gagnerait ainsi à se déprendre de sa connaissance postérieure du dénouement des événements, gardant toujours à l'esprit que les hommes avaient alors un « futur ouvert ${ }^{7} »[$ fig. 16].

Fig. 16 - L'effet de suspension (1.6)

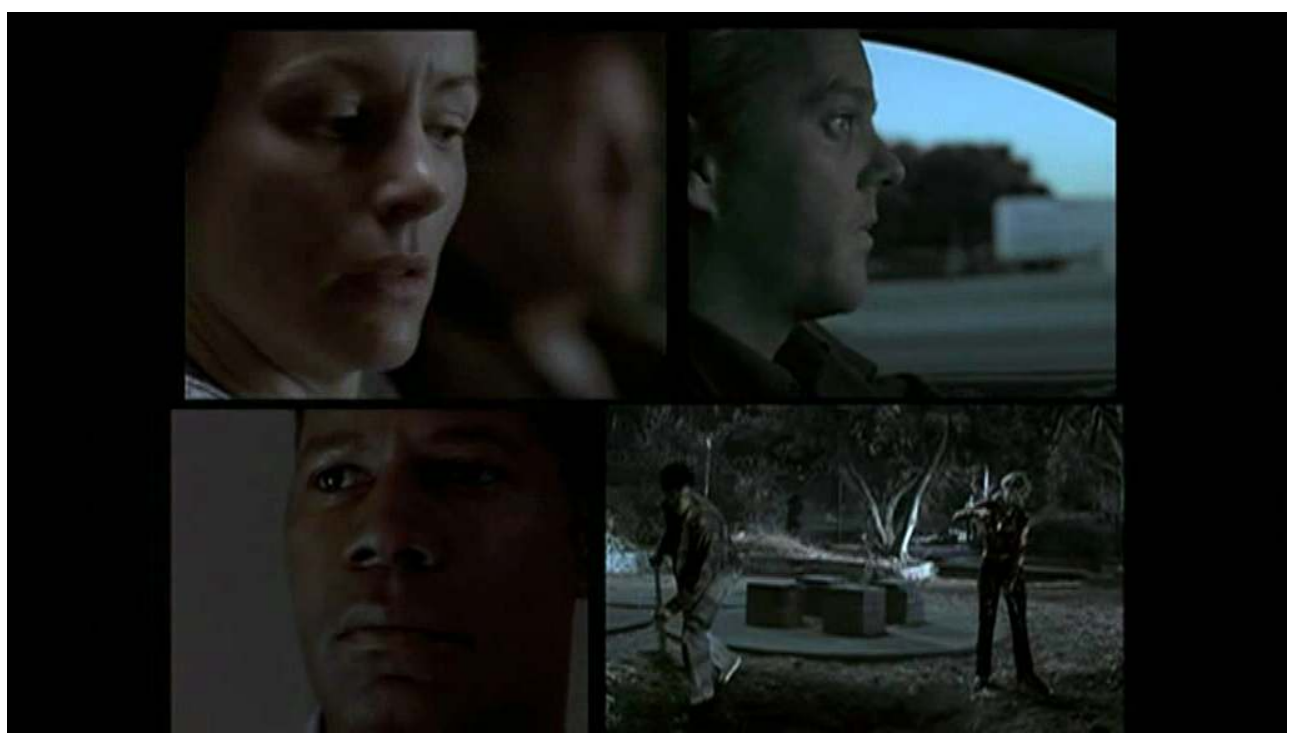

Lorsqu'il surgit au sein d'un épisode, outre les passages obligés précédemment évoqués, que peut signifier le split-screen? Par sa manière d'associer différents personnages en un même cadre, ce procédé offre à la fois une coupe horizontale des enjeux du récit (différentes actions simultanées montrées en temps «réel») et une coupe verticale (révélant la connexion insoupçonnée entre les péripéties d'une adolescente, l'enquête d'agents de terrain anti-terroristes, et en remontant jusqu'au président des États-Unis). 
Le split-screen permet également d'élaborer un discours sur le statut des images, leur flux incessant, leur impact sur nous et sur la question de leur authenticité (voir à ce sujet les films de Brian de Palma). C'est pourquoi se fait jour en permanence, dans les films qui l'utilisent, une conscience profonde des simulacres et des faux-semblants alimentés par l'omniprésence des médias, des écrans et de la circulation des images [fig. 17-18].

Fig. 17-18 - L'omniprésence des médias : L'Affaire Thomas Crown et L'Étrangleur de Boston
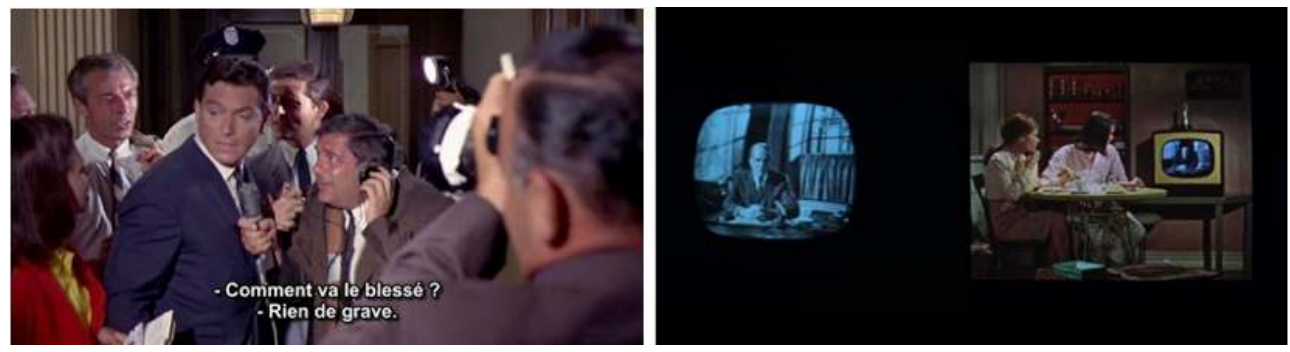

Par ailleurs, le split-screen donne au spectateur l'impression d'assister au déroulement du récit comme s'il se trouvait face à une régie de contrôle vidéo, avec ses multiples écrans, passant de l'un à l'autre [fig. 19].

Fig. 19 - Écrans de contrôle (1.6)

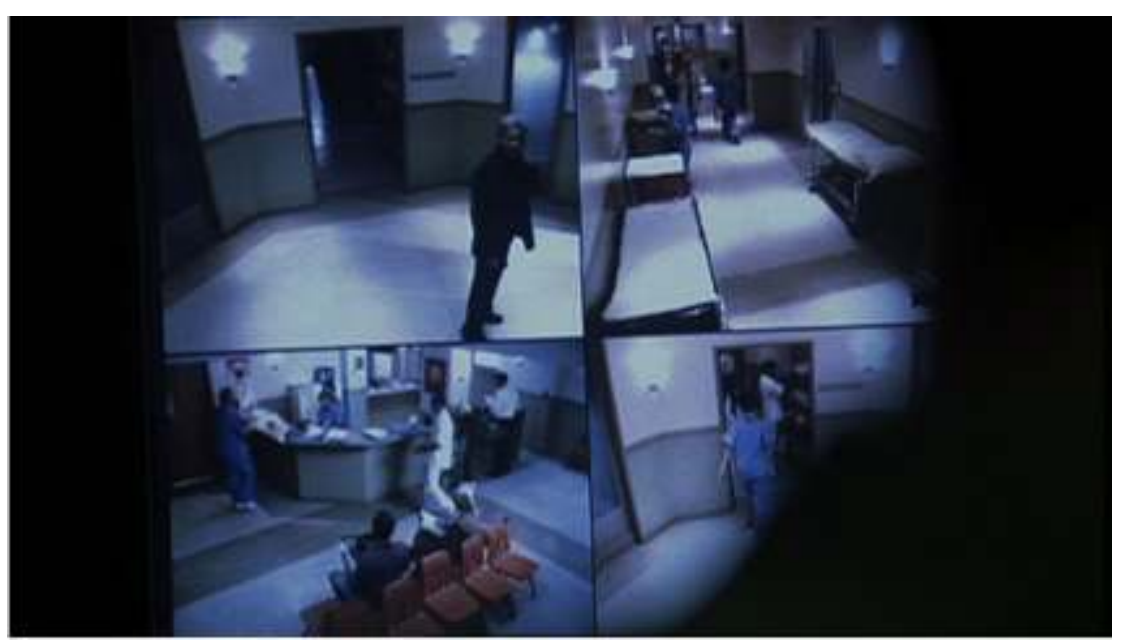

Cette idée renforce le sentiment d'être plongé au cœur d'une société foncièrement quadrillée, surveillée, paranoïaque. En cela, l'emploi du split-screen apparaît opportun, suggérant que chaque individu constitue une petite cellule suspicieuse, irrémédiablement solitaire et ne pouvant se fier qu'à elle-même [fig. 20]. Comme le remarque Jean-Baptiste Thoret :

Parce qu'il expose une image sous toutes ses coutures, le split-screen travaille naturellement à une forme de vision absolue. On comprend alors en quoi le désir de maîtrise et l'obsession du complot constituent ses terrains d'élection les plus fertiles 8 . 
Fig. 20 - Isolement de chacun, suspicion de tous : 24 heures chrono (1.2)

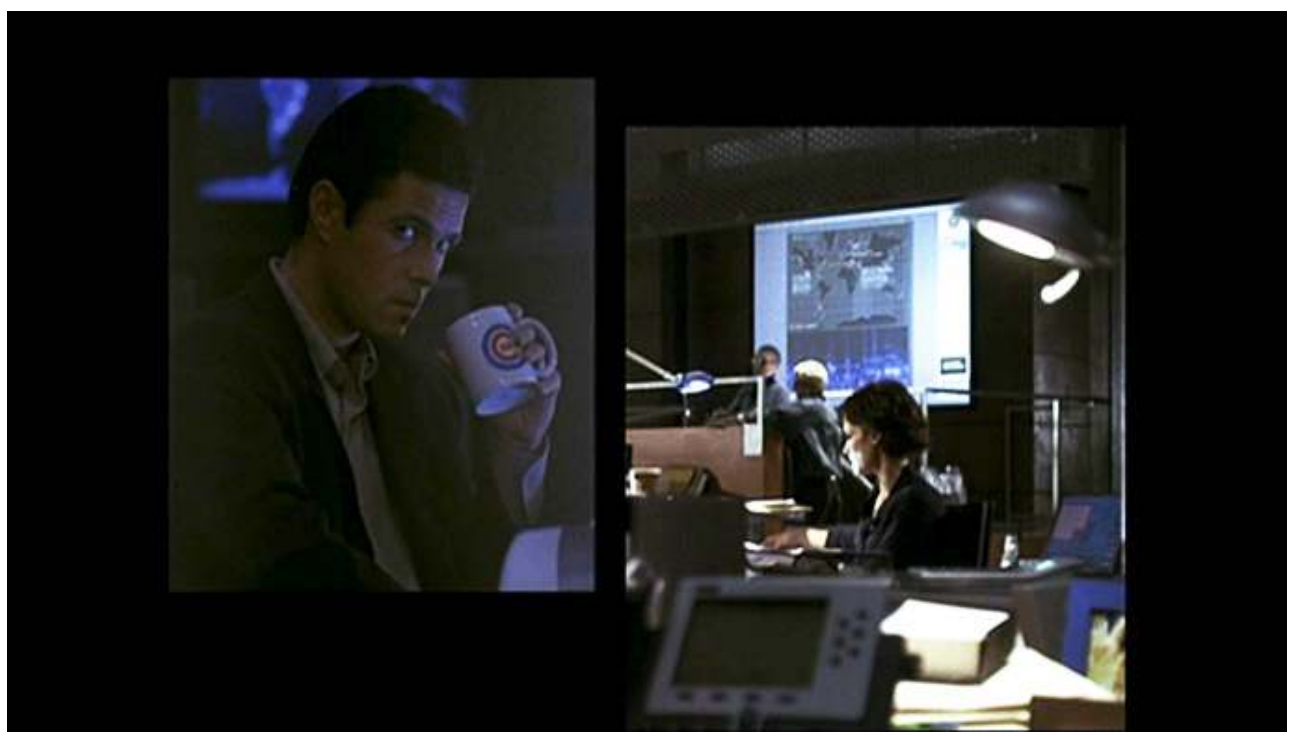

L'usage du split-screen apparaît également opportun si l'on convoque à nouveau André Bazin et sa formule célèbre selon laquelle les limites de l'écran de cinéma ne forment pas un cadre, mais un cache. Selon Bazin, l'écran est centrifuge, il projette notre attention vers le hors-champ. Si bien que la multiplication des cadres dans le cadre s'avère être, en définitive, non pas tant une multiplication des points de vue qu'une multiplication des caches [fig. 21-22].

Fig. 21-22 - L'écran est un cache : L'Affaire Thomas Crown et L'Étrangleur de Boston

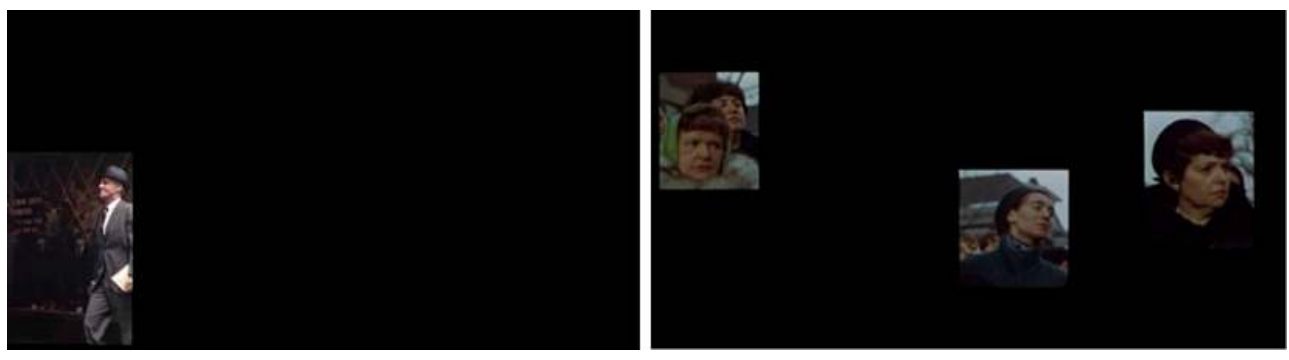

Le split-screen permet donc d'implanter le hors-champ (c'est-à-dire la menace) au sein même du cadre et non plus à ses bords. Nous retrouvons ici les deux angoisses paranoïaques fondatrices de la série 24 heures : d'une part, nous pouvons être pénétrés de partout (la menace terroriste actualise ici une angoisse des plus archaïques); d'autre part, le danger qui nous guette provient également, et peut-être avant tout, de l'intérieur (la taupe).

Enfin, le split-screen visualise le clivage moral de Jack Bauer et des administrations successives (celle de David Palmer notamment) qui ont à mettre en balance le respect de la loi et la nécessité de l'action, la lenteur du protocole général et l'urgence des situations particulières, la morale des principes et celle des conséquences. Si bien que la figuration du clivage (psychique, relationnel, social, moral) est sans aucun doute la grande affaire du split-screen. 


\section{D'un certain sadisme narratif}

27 Une hypothèse. Nous nous plaisons à imaginer le split-screen dans 24 heures comme l'instrument privilégié d'une instance narrative, ou d'un narrateur, discrètement sadique, dont le profil psychologique pourrait correspondre à celui que dresse Nina Myers de l'homme d'affaire Ted Cofell : «Intelligent », « anal », " passif-agressif » « control-freak ${ }^{9}$ ». Ce narrateur omniscient, tout-puissant, jouant sur la distribution et la rétention d'images qu'il manipule comme s'il était face à des écrans de contrôle, semble constamment sélectionner au sein du split-screen le cadre qu'il désire afin de nous livrer l'image voulue en plein écran. Il faudrait faire le lien entre, d'une part, cette instance narrative virtuelle qui commande l'agencement des cadres (ce "Grand Imagier» pour reprendre une expression d'Albert Laffay ${ }^{10}$ ) et, d'autre part, la voix neutre, mystérieuse et feutrée qui annonce le cadre temporel du récit au début de chaque épisode ( The following takes place between 2 am et 3 am... »). Il se trouve que c'est Kiefer Sutherland qui prête sa voix à chaque début d'épisode mais cela n'est pas explicitement dit au spectateur. Et même si, au début des épisodes 8 à 12 de la première saison, Jack Bauer explique qu'il vit la journée la plus longue de sa vie, et même si l'on reconnaît bien la voix du comédien, une ambiguïté demeure sur l'identité de cette « l'horloge parlante » anonyme.

Pourquoi donc avons-nous besoin d'une voix pour donner l'heure ou rappeler que les événements se déroulent en temps réel le jour des primaires? Avec cette voix off mystérieuse, nous avons ici affaire à ce que Michel Chion appelle un acousmêtre, c'est-àdire une voix dont on ignore la provenance et qui, du fait de cette désincarnation, se voit prêter différents pouvoirs : être partout, tout voir, tout savoir, tout pouvoir ${ }^{11}$. C'est une figure incontournable du cinéma américain que cette voix toute-puissante que l'on ne voit pas mais qui, elle, voit tout, vous possède et vous manipule comme un pantin : Die Hard 3 (1995) de John McTiernan, Scream (1996) de Wes Craven, ou Phone Game (2002) de Joel Schumacher (qui se passe entièrement dans une cabine téléphonique depuis laquelle un homme est à la merci d'une voix mystérieuse. Est-ce un hasard si le comédien qui prête sa voix au sniper manipulateur et invisible n'est autre que Kiefer Sutherland ?...). En tant que spectateurs, nous sommes dans 24 heures chrono les jouets de cet acousmêtre, ce narrateur tout-puissant qui dispose des images à sa guise, ce control freak qui s'est imposé à lui-même la règle du temps réel ${ }^{12}$.

Mais en quoi l'usage du split-screen correspondrait-il à une certaine forme de sadisme narratif? Loin d'accroitre notre capacité de vision ou notre intelligibilité du récit en nous offrant une multiplicité de points de vue, le split-screen ne génère au contraire qu'une plus grande confusion. L'image est morcelée en plusieurs vignettes (ce qui en diminue la visibilité), l'espace n'est plus construit par le montage mais déplié (ce qui provoque souvent une perte de repères). Plusieurs scènes se proposent à nous simultanément et il faut toujours une petite seconde d'ajustement pour accoler le son que l'on entend aux images correspondantes. Nous avons donc toujours un peu de retard sur les personnages. Là où le split-screen semblait devoir nous procurer une position de surplomb, de maîtrise sur le récit, il nous met sans cesse à distance en brandissant son patchwork d'écrans qui nous signifie notre position de spectateurs. Oui, le split-screen est peut-être avant tout l'instrument privilégié du sadisme narratif de la série, qui en fait tout le délice, et dont l'exemple le plus parfait est ce chronomètre qui, tout au long de l'épisode, nous rappelle cruellement que le temps de plaisir nous est compté. 


\section{BIBLIOGRAPHIE}

BAZIN, André, « Ontologie de l'image photographique », in Qu'est-ce que le cinéma (1959), Paris, Cerf, 1990, p. 9-17.

- « Montage interdit », in Ibid, p. 49-61.

- «L'évolution du langage cinématographique », in Ibid, p. 63-80.

CHION, Michel, « L’acousmêtre », in La Voix au cinéma, Paris, Cahiers du cinéma, 1982, p. 25-33.

DAUMAS, Alice, Le split screen. Usages de l'écran partagé, de son utilisation ponctuelle à sa radicalisation au sein du cinéma de fiction, Mémoire de fin d'études sous la direction de Benoît Turquety et Vincent Amiel, ENS Louis Lumière, 2009.

EISENSTEIN, Sergueï, « Méthodes de montage » (1929), in Le Film, sa forme, son sens, trad. Armand Panigel, Paris, Christian Bourgois, 1976, p. 63-71.

LAFFAY, Albert, Logique du cinéma. Création et spectacle, Paris, Masson, 1964.

RICCEUR, Paul, « Histoire et mémoire », in Antoine de Baecque et Christian Delage (dir.), De l'histoire au cinéma, Bruxelles, éd. Complexe, 1998, p. 17-28.

SOBIESZCZANSKI, Marcin, MASONI LACROIX, Céline, (dir.), Du Split-Screen Au Multi-Screen : La narration vidéo-filmique spatialement distribuée, Berne, Peter Lang, 2010.

SUROWIECKI, James, « The Worst Day Ever. A 24 writer talks about torture, terrorism, and fudging “real time" » : http://www.slate.com/articles/news_and_politics/ interrogation/2006/01/the_worst_day_ever.html

THORET, Jean-Baptiste, « Le Split-screen », Cahiers du cinéma, hors-série, novembre 2000, p. 84.

TYLSKI, Alexandre, « Le split-screen audiovisuel. Contemporanéité(s) d'une figure », Positif, $\mathrm{n}^{\circ} 566$, avril 2008, p. 56-58.

VANOYE, Francis, (dir.), Dictionnaire de l'image, Paris, Vuibert, 2008

WORMS, Frédéric, Le Moment du soin. À quoi tenons-nous ?, Paris, Presses Universitaires de France, coll. « Éthique et philosophie morale », 2010.

\section{NOTES}

1. Voir notamment l'entrée «Split-screen » du Dictionnaire de l'image publié sous la direction de Francis Vanoye (Paris, Vuibert, 2008). Pour une analyse historique, technique et esthétique de ce procédé, voir notamment: Alexandre Tylski, «Le split-screen audiovisuel. Contemporanéité(s) d'une figure ", Positif, n 566, avril 2008, p. 56-58; Alice Daumas, Le split screen. Usages de l'écran partagé, de son utilisation ponctuelle à sa radicalisation au sein du cinéma de fiction, Mémoire de fin d'études sous la direction de Benoît Turquety et Vincent Amiel, ENS Louis Lumière, 2009 ; Marcin Sobieszczanski et Céline Masoni Lacroix (dir.), Du Split-Screen Au Multi-Screen : La narration vidéofilmique spatialement distribuée, Berne, Peter Lang, 2010.

2. André Bazin, « Montage interdit », in Qu'est-ce que le cinéma (1959), Paris, Cerf, 1990, p. 59. 
3. D'où la prédilection de Bazin pour le plan-séquence et la profondeur de champ. Voir à ce propos, in Op. cit., les articles «Ontologie de l'image photographique » (p. 9-17) et «L'évolution du langage cinématographique » (p. 63-80).

4. Cette idée connaîtra une grande postérité au sein de la réflexion critique française, notamment au tournant des années 1960 dans les colonnes des Cahiers du cinéma, où elle sera réinvestie dans le champ de la morale avec le fameux exemple du travelling (chez des auteurs tels que Luc Moullet, Jean-Luc Godard, Jacques Rivette ou encore Serge Daney).

5. Nous reprenons ici la formule de Frédéric Worms en titre de son ouvrage Le Moment du soin. À quoi tenons-nous ?, Paris, Presses Universitaires de France, coll. «Éthique et philosophie morale », 2010.

6. Lire à ce propos Sergueï Eisenstein, « Méthodes de montage » (1929), in Le Film, sa forme, son sens, trad. Armand Panigel, Paris, Christian Bourgois, 1976, p. 63-71.

7. Paul Ricœur, "Histoire et mémoire ", in Antoine de Baecque et Christian Delage (dir.), De l'histoire au cinéma, Bruxelles, éd. Complexe, 1998, p. 27.

8. Jean-Baptiste Thoret, « Le Split-screen », Cahiers du cinéma, hors-série, novembre 2000, p. 84.

9. 24 heures chrono (1.9)

10. Albert Laffay, Logique du cinéma. Création et spectacle, Paris, Masson, 1964.

11. Michel Chion, «L'acousmêtre ", in La Voix au cinéma, Paris, Cahiers du cinéma, 1982, p. 25-33.

12. Rappelons qu'au commencement était la règle, le producteur Joel Surnow ayant tout d'abord eu l'idée d'une série dont les saisons de vingt-quatre épisodes correspondraient aux vingt-quatre heures d'une seule journée. L'idée de suivre un agent d'une unité anti-terroriste n'est venue qu'après. Lire à ce propos l'entretien donné à Slate.com par Michael Loceff, l'un des principaux scénaristes et producteurs de la série (James Surowiecki, « The Worst Day Ever. A 24 writer talks about torture, terrorism, and fudging "real time"»: http://www.slate.com/articles/ news_and_politics/interrogation/2006/01/the_worst_day_ever.html, consulté le 20 juin 2014).

\section{RÉSUMÉS}

Presque aussi ancien que le cinéma lui-même, le procédé du split-screen, consistant à diviser le cadre filmique en plusieurs portions, compte parmi les figures stylistiques les plus marquantes de 24 heures chrono. Or, les usages qui en sont faits, aussi bien au cinéma que dans la série, apparaissent bien souvent d'une grande pauvreté, le split-screen se résumant à exacerber les modes d'écriture propres au cinéma : ingestion au sein du cadre du montage alterné ou du champ contrechamp, nivellement des raccords, atténuation de la dimension de choix propre au cadrage, aliénation automatique des différentes images à une seule et même temporalité, etc. Pourtant, dans 24 heures chrono, l'utilisation du split-screen présente un intérêt inattendu. À travers le morcellement de l'image et l'altération de sa «lisibilité ", grâce à des effets de confusion et de perte de repères, le split-screen constitue l'un des instruments privilégiés d'un certain sadisme narratif qui contribue grandement à l'originalité de la série et au plaisir qu'elle nous procure.

Almost as old as cinema itself, the split-screen - in which the filmic frame is divided into several parts - is one of the most prominent stylistic figures in 24 , but is often used only to enhance the writing modes that are specific to cinema: alternate editing and shots/reverse shots are swallowed by the frame; link shots are evened out; framing choices are toned down; the different images are automatically reduced to one temporality only, etc. Through the division of images 
and the alteration of their 'readability' with confusing or defamiliarizing effects, the split screen stands as one of the preferred tools to create a certain kind of narrative sadism, which contributes to the series' originality and to the viewing pleasure it provides.

\section{INDEX}

Keywords : split screen, framing, editing, off-camera, aesthetics, narrative sadism, 24 Mots-clés : split-screen, cadrage, montage, hors-champ, esthétique, acousmêtre, sadisme narratif, 24 heures chrono

\section{AUTEUR}

\section{OPHIR LEVY}

Ophir LEVY enseigne l'esthétique et l'histoire du cinéma à l'université Paris III - Sorbonne Nouvelle depuis 2005. Docteur en histoire du cinéma de l'Université Paris I - Panthéon-Sorbonne (sous la direction de Sylvie Lindeperg), sa thèse intitulée Les Images clandestines (prochainement publiée par les éditions Hermann) a reçu le "Prix de la Recherche 2014" décerné par l'Inathèque. Philosophe de formation, il est l'auteur de Penser l'humain à l'aune de la douleur. Philosophie, histoire, médecine. 1845-1945 (L'Harmattan, 2009). 